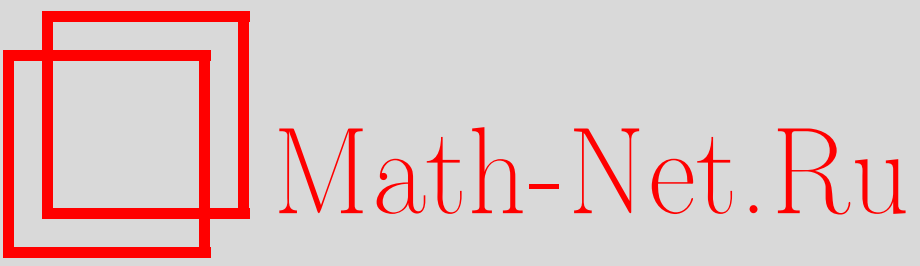

Ю. С. Вернов, М. Н. Мнацаканова, Уайтмановский аксиоматический подход в некоммутативной квантовой теории поля, ТМФ, 2005, том 142, номер 2, 403416

DOI: https://doi.org/10.4213/tmf1791

Использование Общероссийского математического портала Math-Net.Ru подразумевает, что вы прочитали и согласны с пользовательским соглашением http://www.mathnet.ru/rus/agreement

Параметры загрузки:

IP : 3.85 .183 .62

26 апреля 2023 г., $17: 12: 27$ 


\section{УАЙТМАНОВСКИЙ АКСИОМАТИЧЕСКИЙ ПОДХОД В НЕКОММУТАТИВНОЙ КВАНТОВОЙ ТЕОРИИ ПОЛЯ}

Аксиоматический подход, основанный на функциях Уайтмана, развит в некоммутативной квантовой теории поля. Доказано, что основные результаты аксиоматического подхода остаются справедливыми, если некоммутативность затрагивает только пространственные переменные.

Ключевые слова: некоммутативность, квантовая теория поля, аксиоматический подход, функции Уайтмана, локальная коммутативность, спектральность, $C P T$-теорема.

\section{1. ВВЕДЕНИЕ}

Аксиоматический подход, развитый в работах Уайтмана, Йоста, Боголюбова, Хаага и других (см. [1]-[4] и ссылки в них), дал возможность сформулировать квантовую теорию поля как последовательную математически строгую теорию. Этот подход был обобщен на теорию калибровочных полей, прежде всего в работах Моркио и Строкки [5], [6].

В рамках этого подхода на основе общих принципов теории был получен ряд фундаментальных результатов, например, доказаны $C P T$-теорема и теорема о связи спина и статистики. Были выведены аналитические свойства амплитуд рассеяния по энергии и переданному импульсу, послужившие основой для вывода строгих ограничений на высокоэнергетическое поведение этих амплитуд (см. обзор [7]).

В настояшее время широко рассматриваются различные обобшения стандартной квантовой теории поля, в частности некоммутативная квантовая теория поля, в основе которой лежат коммутационные соотношения между координатами

$$
\left[\hat{x}_{\mu}, \hat{x}_{\nu}\right]=i \theta_{\mu \nu},
$$

где $\theta_{\mu \nu}$ - постоянная матрица. Интерес к такого рода теориям имеет давнюю историю [8]. Новый этап в их развитии связан, с одной стороны, с построением некоммутативной геометрии [9], а с другой - с новыми аргументами в пользу такого обобшения

\footnotetext{
${ }^{*}$ Институт ядерных исследований РАН, Москва, Россия. E-mail: vernov@ms2.inr.ac.ru

${ }^{\dagger}$ Научно-исследовательский институт ядерной физики Московского государственного университета, Москва, Россия. E-mail: mnatsak@theory.sinp.msu.ru
} 
теории на сверхмалые расстояния, т.е. сверхвысокие энергии [10]. Кроме того, большой интерес к таким теориям связан с тем, что в ряде случаев они являются низкоэнергетическим пределом струнных теорий [11]. Обзор основных результатов этого направления дан в работах [12], [13].

Некоммутативные теории распадаются естественным образом на два класса: теории, в которых время коммутирует с пространственными переменными, т.е. $\theta_{0 i}=0$, и теории, в которых $\theta_{0 i} \neq 0$ (общий случай). Если $\theta_{0 i}=0$, то в теории нет трудностей с унитарностью и причинностью, характерных для общего случая [14]-[16], хотя и в нем сушествуют варианты последовательной теории [17]. Отметим, что именно вариант с $\theta_{0 i}=0$ является низкоэнергетическим пределом струнной теории [11]. В этом же варианте теории исследовались аналитические свойства амплитуды упругого рассеяния по энергетической и угловой переменным [18]-[22]. В работах [19], [20] были выведены дисперсионные соотношения вперед. В работе [22] доказана справедливость неравенства Фруассара-Мартена.

Бо́льшая часть вычислений в некоммутативной теории поля связана с теорией возмушений. Однако в ряде работ [23]-[27] был развит более обший подход. В частности, идеи алгебраической теории поля были положены в основу исследований в работах [24], [25], а в работах [26], [27] рассмотрение базируется на введении функций Уайтмана. Настоящая статья посвящена развитию последнего подхода.

Как и в работах [26], [27] мы рассматриваем вариант теории, в котором $\theta_{0 i}=0$. В этом случае, не теряя обшности [28], можно выбрать представление алгебры, заданной соотношением (1), таким образом, что только $\theta_{12}=-\theta_{21} \neq 0$. Следовательно, мы имеем две некоммутативные координаты $x_{1}$ и $x_{2}$ и две коммутативные $-x_{0}$ и $x_{3}$. По коммутативным координатам мы имеем, по сушеству, двумерную теорию поля. В частности, функции Уайтмана оказываются аналитическими функциями по коммутативным переменным в области, являюшейся двумерным аналогом соответствуюшей области аналитичности в стандартной теории [3], [26]. Этот вывод основывается на $S O(1,1)$-инвариантности теории по отношению к коммутативным переменным. При этом вместо стандартного условия спектральности [1], [3] используется его аналог только для компонент импульса, соответствуюших коммутативным переменным. А именно, предполагается, что любой базисный вектор в импульсном пространстве является времениподобным по указанным компонентам. Вывод некоммутативных аналогов основных результатов аксиоматического подхода, таких как С $P T$-теорема и теорема о связи спина и статистики, основывается на следуюшем аналоге условия локальной коммутативности [28]:

$$
[\varphi(\hat{x}), \varphi(\hat{y})]=0
$$

если $\left(x_{0}-y_{0}\right)^{2}-\left(x_{3}-y_{3}\right)^{2}<0$.

Что касается некоммутативных переменных, то достаточно предполагать инвариантность теории по отношению к группе отражений некоммутативных координат $I_{s}(2)$. Таким образом, для нас важно лишь сушествование $S O(1,1) \otimes I_{s}(2)$-симметрии, а также аналогов условий локальной коммутативности и спектральности. Более того, ряд результатов основывается только на $S O(1,1)$-симметрии и условии спектральности. 
В настоящей работе в некоммутативной теории будут введены функции Уайтмана в общем виде (10). Частными случаями этих функций являются функции, рассматривавшиеся в работе [26] (если в них перейти к мойаловскому произведению в совпадающих точках), и функции, введенные в работе [27]. Далее для комплексного скалярного поля будет доказан ряд классических результатов аксиоматической квантовой теории поля, в частности, будет доказана эквивалентность условия локальной коммутативности и симметрии функций Уайтмана по отношению к перестановке аргументов в области аналитичности. Будет доказана $C P T$-теорема для комплексного поля и введены классы эквивалентности Борхерса ${ }^{1)}$. Мы рассмотрим также теорему о связи спина и статистики.

Подчеркнем одно сушественное обстоятельство. Принципиальную роль во всем проводимом ниже рассмотрении играет сушествование аналогов условия локальной коммутативности и условия спектральности. Эти аналоги справедливы и в коммутативной теории. Таким образом, оказывается, что в коммутативной теории для воспроизведения основных результатов аксиоматического подхода достаточно исходить из более слабых предположений, чем стандартные. Некоммутативность играет роль новой физической реальности, делающей эти ослабления условий локальной коммутативности и спектральности неизбежными. Разумеется, и в других обобщениях стандартной теории, в которых справедливы указанные аналоги условий локальной коммутативности и спектральности, полученные в статье результаты остаются справедливыми.

Статья построена следующим образом. Поскольку развиваемый подход является естественным обобшением стандартного формализма, мы изложим его суть. Затем в некоммутативном случае будет рассмотрена связь между функциями Уайтмана в некоммутативном и коммутативном пространствах и сформулированы аналоги условия локальной коммутативности и спектральности ${ }^{2)}$. Они играют ключевую роль в распространении классических результатов аксиоматического подхода на некоммутативную теорию. Прежде всего будет показано, что трансляционная инвариантность вместе с $S O(1,1)$-симметрией ведет к невозможности описания теории операторами, заданными в точке. Далее будет показано, что в обшем случае так же, как и в случаях, рассмотренных в работах [26], [27], функции Уайтмана аналитичны по коммутирующим переменным в области, содержащей подмножество точек Йоста. Будет показано, что условие локальной коммутативности позволяет расширить область аналитичности функций Уайтмана и что, как и в коммутативном случае, оно эквивалентно симметрии функций Уайтмана в области аналитичности. Это обстоятельство приводит к невозможности простого ослабления условия локальной коммутативности (см. утверждение 2).

Далее в случае комплексного скалярного поля будет доказана С будет рассмотрено условие взаимной слабой локальной коммутативности двух полей и доказано, что это условие эквивалентно наличию у этих полей одного и того же оператора $C P T$-преобразования. Это обстоятельство позволяет ввести в некоммутативном

\footnotetext{
1) Близкие результаты, связанные с построением классов эквивалентности Борхерса, были получены в работе [29], вышедшей после направления в печать текста этой статьи.

2) Ниже, говоря об этих условиях, мы опускаем слово "аналог".
} 
случае классы эквивалентности Борхерса.

Заключительная часть работы посвящена теореме о связи спина и статистики.

\section{2. ОСНОВНЫЕ ПОСТУЛАТЫ}

Прежде всего напомним суть уайтмановского подхода в квантовой теории поля. Чтобы не усложнять формулы, мы сформулируем этот подход так, как если бы поле $\varphi(x)$ было задано в точке. В действительности операторами являются величины

$$
\varphi_{f} \equiv \int \varphi(x) f(x) d x
$$

где $f(x)$ - некоторая пробная функция [1]-[3]. Предполагается, что вакуумный вектор $\Psi_{0}$ является циклическим, иными словами, предполагается, что в рассматриваемом пространстве $J$ векторы типа

$$
\varphi^{\sigma_{1}}\left(x_{1}\right) \varphi^{\sigma_{2}}\left(x_{2}\right) \ldots \varphi^{\sigma_{n}}\left(x_{n}\right) \Psi_{0}
$$

образуют базис. Значок $\sigma_{i}$ означает, что $\varphi^{\sigma_{i}}\left(x_{i}\right)$ может быть как $\varphi\left(x_{i}\right)$, так и $\varphi^{+}\left(x_{i}\right)$, где $\varphi^{+}\left(x_{i}\right)$ - сопряженный оператор. При этом, если $\varphi^{\sigma_{i}}\left(x_{i}\right)=\varphi\left(x_{i}\right)$, то $\varphi^{\sigma_{i}^{+}}\left(x_{i}\right)=$ $\varphi^{+}\left(x_{i}\right)$, и наоборот. Мы принимаем, что в пространстве $J$ задано скалярное произведение $\langle\cdot, \cdot\rangle$, которое может быть индефинитным. Условие цикличности вакуума означает, что каждый вектор пространства $J$ может быть аппроксимирован с любой точностью векторами типа (4). Мы не уточняем это условие, поскольку для дальнейшего сушественно только то, что для любых векторов скалярное произведение может быть аппроксимировано с произвольной точностью линейной комбинацией функций Уайтмана

$$
W\left(x_{1}, x_{2}, \ldots, x_{n}\right)=\left\langle\Psi_{0}, \varphi^{\sigma_{1}}\left(x_{1}\right) \varphi^{\sigma_{2}}\left(x_{2}\right) \ldots \varphi^{\sigma_{n}}\left(x_{n}\right) \Psi_{0}\right\rangle .
$$

Очевидно, что скалярное произведение двух базисных векторов есть некоторая функция Уайтмана.

Переходя к некоммутативному пространству, можно ввести

$$
W\left(\hat{x}_{1}, \hat{x}_{2}, \ldots, \hat{x}_{n}\right)=\left\langle\Psi_{0}, \varphi^{\sigma_{1}}\left(\hat{x}_{1}\right) \varphi^{\sigma_{2}}\left(\hat{x}_{2}\right) \ldots \varphi^{\sigma_{n}}\left(\hat{x}_{n}\right) \Psi_{0}\right\rangle .
$$

Та же самая функция Уайтмана может быть записана и в коммутативном пространстве при соответствуюшем изменении произведения операторов.

Рассмотрим сначала случай, когда $x_{i}=x$ для любого $i$. В некоммутативной теории, как известно [12], [13], произведение двух произвольных операторов $\varphi(x)$ и $\psi(x)$ заменяется $\star$-произведением (мойаловским произведением)

$$
\phi(\hat{x}) \psi(\hat{x}) \rightarrow \phi(x) \star \psi(x),
$$

где

$$
\phi(x) \star \psi(x)=\left.\phi(x) \exp \left(\frac{i}{2} \theta_{\mu \nu} \frac{\overleftarrow{\partial}}{\partial x^{\mu}} \frac{\vec{\partial}}{\partial y^{\nu}}\right) \psi(y)\right|_{x=y}
$$

Соответственно

$$
W(\hat{x}, \hat{x}, \ldots, \hat{x}) \rightarrow W_{\star}(x, x, \ldots, x)=\left\langle\Psi_{0}, \varphi^{\sigma_{1}}(x) \star \varphi^{\sigma_{2}}(x) \star \cdots \star \varphi^{\sigma_{n}}(x) \Psi_{0}\right\rangle .
$$


ЗАмЕчАниЕ 1 . Вследствие трансляционной инвариантности $W_{\star}(x, x, \ldots, x)$ в действительности не зависит от $x$.

Рассмотрим теперь противоположный случай, когда все соседние $x_{i}$ различны. Очевидно, что существует большая неопределенность в выборе соответствующего обобщения мойаловского произведения для различных точек. Замечательно, что возможность распространения аксиоматического подхода на некоммутативную теорию не зависит от конкретного вида этого обобщения, если только оно удовлетворяет некоторым общим требованиям. Итак, если $x_{i} \neq x_{i \pm 1}$ для всех $i$, запишем функцию Уайтмана в следуюшем общем виде:

$$
W_{\tilde{\star}}\left(x_{1}, x_{2}, \ldots, x_{n}\right)=\left\langle\Psi_{0}, \varphi^{\sigma_{1}}\left(x_{1}\right) \tilde{\star} \varphi^{\sigma_{2}}\left(x_{2}\right) \tilde{\star} \cdots \tilde{\star} \varphi^{\sigma_{n}}\left(x_{n}\right) \Psi_{0}\right\rangle .
$$

Как и в обычном случае, мы предполагаем, что векторы типа

$$
\varphi^{\sigma_{1}}\left(x_{1}\right) \tilde{\star} \varphi^{\sigma_{2}}\left(x_{2}\right) \tilde{\star} \cdots \tilde{\star} \varphi^{\sigma_{n}}\left(x_{n}\right) \Psi_{0}
$$

образуют набор базисных векторов в рассматриваемом пространстве $J_{\tilde{夭}}$, а $\Psi_{0}$ - циклический вектор.

ЗАМЕЧАнИЕ 2. Естественно считать, что вследствие трансляционной инвариантности $\varphi^{\sigma_{x}}(x) \Psi_{0}=\varphi^{\sigma_{x}}(x) \tilde{\star} \Psi_{0}$ индекс $\sigma_{x}$ имеет тот же смысл, что и $\sigma_{i}$.

Из выражения (11) и замечания 2 непосредственно следует, что оператором в $J_{\tilde{\star}}$ является не $\varphi^{\sigma_{x}}(x)$, а только комбинация $\varphi^{\sigma_{x}}(x) \tilde{\star}$. Этот факт отражает нелокальную природу некоммутативной теории.

Перейдем теперь к случаю, когда $x_{i}=x_{i+1}$ для некоторых $i$. Очевидно, что в совпадающих точках $\tilde{\star}=\star$. Отметим, что вследствие трансляционной инвариантности функции Уайтмана являются в действительности функциями разностей координат $\xi_{i}=x_{i}-$ $x_{i+1}$. Простейший выбор $\approx$ есть $\tilde{\star}=1$ для различных соседних точек. Этот выбор фактически сделан в работе [26]. Отметим, однако, что в этой работе обычное произведение операторов используется и в совпадаюших точках, что, строго говоря, соответствует коммутативной теории с $S O(1,1) \otimes S O(2)$-инвариантностью. Другим естественным выбором $\tilde{\star}$-произведения является непосредственное обобщение мойаловского произведения на различные точки [13], т.е. $\tilde{\star}=\star$. Такой выбор соответствует функциям Уайтмана, введенным в работе [27]. В этом случае некоммутативность проявляется не только в совпадаюших точках, но и в их окрестности. При этом мы автоматически приходим к правильному выражению в совпадающих соседних точках. Чтобы избежать проблемы проявления некоммутативности на больших расстояниях, мы можем умножить $W_{\star}\left(\xi_{1}, \ldots, \xi_{n-1}\right)$ на произведение функций $f_{i}\left(\xi_{i}\right)$, нормированных условием $f_{i}(0)=1$ и быстро спадающих вне области некоммутативности, характеризующейся величинами $\theta_{\mu \nu}$.

Безусловно, вопрос о конкретном виде ₹-произведения является существенным. Возможно, построение аксиоматического варианта теории возмушений подобно тому, как это было сделано в коммутативном случае [3], [30], окажется полезным для прояснения этого вопроса. Вместе с тем еше раз подчеркнем, что наши результаты базируются не 
на определенном выборе $\tilde{\star}$-произведения, а на условиях локальной коммутативности и спектральности. Согласно первому из них

$$
\left[\varphi^{\sigma_{x}}(x), \varphi^{\sigma_{y}}(y)\right]=0
$$

если точки $x$ и $y$ разделены пространственноподобным интервалом $(x-y)^{2}<0$. Согласно условию спектральности

$$
W\left(P_{1}, \ldots, P_{n-1}\right)=\frac{1}{(2 \pi)^{2(n-1)}} \int e^{i P_{j} \xi_{j}} W\left(\xi_{1}, \ldots, \xi_{n-1}\right) d \xi_{1} \ldots d \xi_{n-1} \neq 0
$$

только если $P_{i} \in \bar{V}^{+}$для любого $i$, т.е. если $P_{i}^{0} \geqslant\left|\vec{P}_{i}\right|$. Условие спектральности - следствие отсутствия тахионов в системе базисных векторов в импульсном пространстве (см. [1], глава 2).

Условие локальной коммутативности (12) заведомо выполнено, если $\left(x_{0}-y_{0}\right)^{2}-\left(x_{3}-\right.$ $\left.y_{3}\right)^{2}<0$. Вследствие коммутативности координат $x_{0}$ и $x_{3}$ естественно принять, что и в некоммутативном случае [28]

$$
\left[\tilde{\varphi}^{\sigma_{x}}(x), \tilde{\varphi}^{\sigma_{y}}(y)\right]=0
$$

при $x \sim y$, где $\tilde{\varphi}^{\sigma_{x}}(x) \equiv \varphi^{\sigma_{x}}(x) \tilde{\star}$. Здесь и ниже $x \sim y$ означает, что $\left(x_{0}-y_{0}\right)^{2}-\left(x_{3}-\right.$ $\left.y_{3}\right)^{2}<0$.

Соответственно условие спектральности в некоммутативном случае видоизменяется следующим образом:

$$
W\left(P_{1}, \ldots, P_{n-1}\right)=0
$$

если сушествует $i$ такое, что $P_{i} \notin \bar{V}_{2}^{+}$. Здесь $P_{i} \in \bar{V}_{2}^{+}$означает, что $P_{i}^{0} \geqslant\left|P_{i}^{3}\right|$.

Сформулируем теперь условие сопряжения операторов $\tilde{\varphi}^{\sigma_{x}}(x)$. Обратимся к картине в некоммутативном пространстве. Пусть $\Phi-$ произвольный базисный вектор

$$
\Phi=\varphi^{\sigma_{1}}\left(\hat{x}_{1}\right) \ldots \varphi^{\sigma_{n}}\left(\hat{x}_{n}\right) \Psi_{0}
$$

По определению

$$
\langle\Phi, \Psi\rangle=\left\langle\Psi_{0}, \varphi^{\sigma_{n}^{+}}\left(\hat{x}_{n}\right) \ldots \varphi^{\sigma_{1}^{+}}\left(\hat{x}_{1}\right) \Psi\right\rangle
$$

где $\Psi$ - любой вектор. Учитывая соответствие между базисными векторами в некоммутативном и коммутативном пространствах (см. (11)), приходим к выводу, что

$$
[\varphi(x) \tilde{\star}]^{+}=\varphi^{+}(x) \tilde{\star}, \quad\left[\varphi^{+}(x) \tilde{\star}\right]^{+}=\varphi(x) \tilde{\star},
$$

т.e.

$$
\left[\tilde{\varphi}^{\sigma_{x}}(x)\right]^{+}=\tilde{\varphi}^{\sigma_{x}^{+}}(x)
$$

Условия (15)-(17) являются основой для получаемых ниже результатов. 


\section{3. НЕВОЗМОЖНОСТЬ ОПИСАНИЯ ТЕОРИИ ОПЕРАТОРАМИ, ЗАДАННЫМИ В ТОЧКЕ}

Покажем, что, несмотря на то что лоренц-инвариантность нарушена до $S O(1,1)$, оставшейся инвариантности достаточно для исключения возможности описания теории операторами, заданными в точке.

УТВеРЖДЕНИЕ 1. Если $\tilde{\varphi}(x)$ - оператор, заданный в точке, то

$$
W(\xi) \equiv\left\langle\Psi_{0}, \tilde{\varphi}(x) \tilde{\varphi}(y) \Psi_{0}\right\rangle, \quad \xi=x-y,
$$

не зависит от $\xi_{0}$ и $\xi_{3}$.

Используя трансляционную инвариантность и полноту системы векторов $\Psi_{p_{n}, n}$, где $p_{n}$ - импульс, а мультииндекс $n$ содержит все другие характеристики данного состояния, легко получить, что $W(\xi)$ может быть представлена в виде (см. [3], глава 3 )

$$
W(\xi)=\int e^{i p \xi} \varrho(p) d p
$$

где $\varrho(p)>0$. Кроме того, $\varrho(\Lambda, p)=\varrho(p)$ для всех $\Lambda \in S O(1,1)$.

ЗАмЕчАниЕ 3 . Если $J_{\tilde{x}}-$ пространство с индефинитной метрикой, формула (18) справедлива для операторов, принадлежаших дефинитному, например, физическому подпространству.

Положив $\xi=0$, получим

$$
W(0)=\int \varrho(p) d p
$$

Теперь легко видеть, что вследствие положительности $\varrho(p)$ и ее инвариантности относительно преобразований, принадлежащих $S O(1,1)$, интеграл в (19) сходится, только если $\varrho(p)=\delta\left(p_{0}\right) \delta\left(p_{3}\right) \tilde{\varrho}(p)$. Действительно, в противном случае $\varrho(p) \neq 0$, когда $p \in O$, где $O$ - некоторая конечная область, не содержашая точку $p_{0}=0, p_{3}=0$. Применяя к области $O$ преобразования, принадлежащие $S O(1,1)$, и пользуясь инвариантностью и положительностью $\varrho(p)$, можно получить, что интеграл в (19) ограничен снизу бесконечной суммой одинаковых и положительных интегралов, т.е. расходится. Детали доказательства аналогичны соответствуюшим в коммутативном случае [3].

Возврашаясь к формуле (18), сразу получим требуемый результат. Поскольку независимость $W(\xi)$ от $\xi_{0}$ и $\xi_{3}$ бессмысленна с физической точки зрения, то, как и коммутативном случае (см. (3)), нужно перейти от оператора $\tilde{\varphi}(x)$ к оператору

$$
\tilde{\varphi}_{f} \equiv \int \tilde{\varphi}(x) f(x) d x .
$$

Исследование класса пробных функций $f(x)$ выходит за рамки настоящей статьи. Отметим только, что он должен содержать финитные функции по коммутирующим переменным $x_{0}$ и $x_{3}$, чтобы условие (14) могло быть корректно сформулировано. Действительно, в этом случае, если $O \sim O^{\prime}$, т.е. если любая точка области $O$ пространственноподобна любой точке области $O^{\prime}$, условие (14) заменяется следуюшим:

$$
\left[\tilde{\varphi}_{f}, \tilde{\varphi}_{g}\right]=0
$$


для всех $f \in O, g \in O^{\prime}$.

Чтобы упростить формулы, мы рассматриваем ниже формальный оператор $\tilde{\varphi}(x)$ вместо $\tilde{\varphi}_{f}$. При этом суть доказательств, а следовательно и результатов, не меняется. Отметим, что теорию легко сформулировать корректно, поступая так же, как при формулировке обычной теории.

\section{4. АНАЛИТИЧЕСКИЕ СВОЙСТВА ФУНКЦИЙ УАЙТМАНА}

В некоммутативном случае функции Уайтмана аналитичны только по коммутативным переменным $x_{0}$ и $x_{3}[26],[27]$. Отметим, что эти свойства связаны с условием спектральности и $S O(1,1)$-инвариантностью теории. Они не зависят от конкретного вида ₹-произведения, поскольку некоммутируюшие переменные остаются вешественными. Они не зависят также от размерности пространства, а именно, оно может содержать произвольное число некоммутируюших координат. Из условия спектральности (15) непосредственно следует аналитичность функций Уайтмана $W\left(\mu_{1}, \ldots, \mu_{n-1}\right), \mu_{i}=\xi_{i}-$ $i \eta_{i}$, в области $T_{n}^{-}$, которая определена условиями

$$
\eta_{i} \in V_{2}^{+}
$$

для всех $i$. Действительно, поскольку

$$
W\left(\mu_{1}, \ldots, \mu_{n-1}\right)=\frac{1}{(2 \pi)^{2(n-1)}} \int e^{-i P_{j} \xi_{j}-P_{j} \eta_{j}} W\left(P_{1}, \ldots, P_{n-1}\right) d P_{1} \ldots d P_{n-1},
$$

то условие (15) гарантирует сходимость рассматриваемого интеграла, если выполнено условие (21). Подчеркнем, что $\eta_{j}=\left\{\eta_{j}^{0}, 0,0, \eta_{j}^{3}\right\}$. Вследствие аналитичности $W\left(\mu_{1}, \ldots, \mu_{n-1}\right)$ так же, как и $W\left(\xi_{1}, \ldots, \xi_{n-1}\right)$, удовлетворяет $S O(1,1)$-инвариантности. Далее можно применить теорему Баргмана-Холла-Уайтмана [1]-[3], используя комплексную группу $S_{\mathrm{c}} O(1,1)[26]$. В результате область аналитичности $T_{n}^{-}$расширяется до $T_{n}$, где

$$
T_{n}=\cup \Lambda_{\mathrm{c}} T_{n}^{-}, \quad \Lambda_{\mathrm{c}} \in S_{\mathrm{c}} O(1,1) .
$$

Как и в коммутативном случае, $T_{n}$ содержит вешественные точки аналитичности $\tilde{\xi}_{i}=$ $\tilde{x}_{i}-\tilde{x}_{i+1}, \tilde{x}_{i} \in \mathbb{R}-$ точки Йоста. При этом $\tilde{x}_{i} \sim \tilde{x}_{j}$ для всех $i, j$. Отметим, что точки Йоста полностью определяют функции Уайтмана, т.е. функции Уайтмана, совпадаюшие в этих точках, совпадают тождественно.

\section{5. СВЯЗЬ МЕЖКДУ СИММЕТРИЕЙ ФУНКЦИЙ УАЙТМАНА И УСЛОВИЕМ ЛОКАЛЬНОЙ КОММУТАТИВНОСТИ}

Вследствие условия локальной коммутативности (14)

$$
W\left(\tilde{x}_{1}, \ldots, \tilde{x}_{n}\right)=W \Pi\left(\tilde{x}_{1}, \ldots, \tilde{x}_{n}\right),
$$


где $\Pi\left(\tilde{x}_{1}, \ldots, \tilde{x}_{n}\right)$ - произвольная перестановка точек $\tilde{x}_{i}$. Это условие означает, что $W\left(z_{1}, \ldots, z_{n}\right), z_{i}=x_{i}+i y_{i}$, является симметричной функцией своих аргументов в области, получаемой из $T_{n}$ перестановкой точек $\tilde{x}_{i}$ и последуюшим применением преобразований из $S_{\mathrm{c}} O(1,1)$. Таким образом, условие локальной коммутативности позволяет дальше расширить область аналитичности функций Уайтмана.

Покажем, что и, наоборот, условие локальной коммутативности следует из симметрии функций Уайтмана в области аналитичности. Для этого достаточно доказать, что

$$
W\left(z_{1}, \ldots, z_{i-1}, \tilde{x}_{i}, \tilde{x}_{i+1}, z_{i+2}, \ldots, z_{n}\right)=W\left(z_{1}, \ldots, z_{i-1}, \tilde{x}_{i+1}, \tilde{x}_{i}, z_{i+2}, \ldots, z_{n}\right)
$$

где $\tilde{x}_{i}$ и $\tilde{x}_{i+1}$ - точки Йоста, а $z_{j} \in T_{n}^{-}$. Действительно, в равенстве $(25)$ можно перейти к пределу $\operatorname{Im} z_{j}=0$. Для доказательства (25) заметим, что вследствие симметрии функций Уайтмана в области $T_{n}^{-}$справедливо равенство

$$
W\left(z_{1}^{\prime}, \ldots, z_{i}^{\prime}, z_{i+1}^{\prime}, \ldots, z_{n}^{\prime}\right)=W\left(z_{1}^{\prime}, \ldots, z_{i+1}^{\prime}, z_{i}^{\prime}, \ldots, z_{n}^{\prime}\right)
$$

Требуемое равенство будет доказано, если существует набор точек $z_{j}^{\prime}$ и преобразование $\Lambda \in S_{\mathrm{c}} O(1,1)$, переводящее точки $z_{j}^{\prime}$ в $z_{j}, j \neq i, i+1$, а точки $z_{i}^{\prime}, z_{i+1}^{\prime}-$ в $\tilde{x}_{i}$ и $\tilde{x}_{i+1}$. Соответствуюшее преобразование аналогично существующему в коммутативной теории (см. [3], глава 5).

Таким образом, как и к коммутативном случае, условие локальной коммутативности эквивалентно симметрии функций Уайтмана в области аналитичности. Из этой эквивалентности следует невозможность простого обобшения условия локальной коммутативности, а именно справедливо

УТВЕРЖДЕНИЕ 2. Если

$$
\left[\tilde{\varphi}^{\sigma_{x}}(x), \tilde{\varphi}^{\sigma_{y}}(y)\right]=0
$$

при $\left(x_{0}-y_{0}\right)^{2}-\left(x_{3}-y_{3}\right)^{2}<-l^{2}$ и справедливы обычные аксиомы, то выполнено условие локальной коммутативности.

Действительно, при доказательстве аналитичности функций Уайтмана условие локальной коммутативности не используется, и мы имеем то же множество точек Йоста, что и раньше. Рассмотрев подмножество точек Йоста, удовлетворяюшее условию (27), используем это условие для доказательства симметрии функций Уайтмана относительно перестановок аргументов. Остается воспользоваться тем, что локальная коммутативность - следствие симметрии функций Уайтмана. Отметим, что в коммутативном случае утверждение 2 доказано также и при более общих условиях [31].

\section{6. $С Р Т$-ТЕОРЕМА И КЛАССЫ ЭКВИВАЛЕНТНОСТИ БОРХЕРСА}

Доказательство CPT-теоремы в случае комплексного поля является обобшением доказательства для эрмитового поля. Поэтому остановимся только на необходимых изменениях. По определению оператор $C P T$-преобразования определен равенством

$$
\Theta \tilde{\varphi}^{\sigma_{x}}(x) \Theta^{-1}=\tilde{\varphi}^{\sigma_{x}^{+}}(-x) .
$$


Согласно теореме Вигнера (см. [1]), теория будет $C P T$-инвариантна, если $\Theta$ антиунитарен, т.е. если

$$
\langle\Theta \Phi, \Theta \Psi\rangle=\langle\Psi, \Phi\rangle
$$

для любых $\Psi$ и $\Phi$. Поскольку все скалярные произведения базисных векторов (4) сводятся к функциям Уайтмана, достаточно найти условие, при котором

$$
\left\langle\Theta \Psi_{0}, \Theta \tilde{\varphi}^{\sigma_{1}}\left(x_{1}\right) \ldots, \tilde{\varphi}^{\sigma_{n}}\left(x_{n}\right) \Psi_{0}\right\rangle=\overline{\left\langle\Psi_{0}, \tilde{\varphi}^{\sigma_{1}}\left(x_{1}\right) \ldots, \tilde{\varphi}^{\sigma_{n}}\left(x_{n}\right) \Psi_{0}\right\rangle},
$$

где черта означает комплексное сопряжение. Используя определение (28) и условие (17), легко получить, что равенство (29) будет выполнено, если

$$
\left\langle\Psi_{0}, \tilde{\varphi}^{\sigma_{1}}\left(x_{1}\right) \ldots, \tilde{\varphi}^{\sigma_{n}}\left(x_{n}\right) \Psi_{0}\right\rangle=\left\langle\Psi_{0}, \tilde{\varphi}^{\sigma_{n}}\left(-x_{n}\right) \ldots, \tilde{\varphi}^{\sigma_{1}}\left(-x_{1}\right) \Psi_{0}\right\rangle .
$$

Согласно $C P T$-теореме равенство (30) эквивалентно условию слабой локальной коммутативности

$$
\left\langle\Psi_{0}, \tilde{\varphi}^{\sigma_{1}}\left(\tilde{x}_{1}\right) \ldots \tilde{\varphi}^{\sigma_{n}}\left(\tilde{x}_{n}\right) \Psi_{0}\right\rangle=\left\langle\Psi_{0}, \tilde{\varphi}^{\sigma_{n}}\left(\tilde{x}_{n}\right) \ldots \tilde{\varphi}^{\sigma_{1}}\left(\tilde{x}_{1}\right) \Psi_{0}\right\rangle, \quad \tilde{x}_{i} \sim \tilde{x}_{j} .
$$

Это условие очевидным образом вьполняется, если справедливо условие локальной коммутативности. Подчеркнем, однако, что известны примеры полей [3], удовлетворяющих условию слабой локальной коммутативности, но не локальной коммутативности. Поскольку доказательство эквивалентности условий (30) и (31) может быть проведено тем же способом, что и аналогичное доказательство для эрмитова поля [26], [27], мы на нем не останавливаемся. Отметим только, что при этом существенно используется $I_{s}(2)$-инвариантность теории по отношению к некоммутативным координатам. В обычном случае инвариантность функций Уайтмана относительно комплексных преобразований Лоренща включает в себя инвариантность относительно изменения знаков всех координат. В некоммутативном случае $S_{\mathrm{c}} O(1,1)$-инвариантность означает соответствуюшую инвариантность только относительно изменения знака у коммутативных координат. Инвариантность функций Уайтмана относительно изменения знака у некоммутативных координат следует из $I_{s}(2)$-инвариантности.

Найдем теперь условия, при которых два оператора $\tilde{\varphi}(x)$ и $\tilde{\psi}(x) \equiv \psi(x) \tilde{\star}$ имеют обший оператор $C P T$-преобразования. Покажем, что, как и в коммутативном случае, это имеет место, если

$$
\begin{aligned}
& \left\langle\Psi_{0}, \tilde{\varphi}^{\sigma_{1}}\left(\tilde{x}_{1}\right) \ldots \tilde{\varphi}^{\sigma_{i}}\left(\tilde{x}_{i}\right) \tilde{\psi}^{\sigma_{x}}(x) \tilde{\varphi}^{\sigma_{i+1}}\left(\tilde{x}_{i+1}\right) \ldots, \tilde{\varphi}^{\sigma_{n}}\left(\tilde{x}_{n}\right) \Psi_{0}\right\rangle= \\
& \quad=\left\langle\Psi_{0}, \tilde{\varphi}^{\sigma_{n}}\left(\tilde{x}_{n}\right) \ldots \tilde{\varphi}^{\sigma_{i+1}}\left(\tilde{x}_{i+1}\right) \tilde{\psi}^{\sigma_{x}}(x) \tilde{\varphi}^{\sigma_{i}}\left(\tilde{x}_{i}\right) \ldots \tilde{\varphi}^{\sigma_{1}}\left(\tilde{x}_{1}\right) \Psi_{0}\right\rangle
\end{aligned}
$$

при $\tilde{x}_{i} \sim \tilde{x}_{j}, x \sim \tilde{x}_{j}$ для всех $i, j$. Предполагается, что поле $\tilde{\psi}^{\sigma_{x}}(x)$ удовлетворяет условиям, при которых функции в (32) аналитичны в той же области, что и функции Уайтмана поля $\tilde{\varphi}^{\sigma_{x}}(x)$, однако $\Psi_{0}$ может не быть циклическим вектором для полей $\tilde{\psi}^{\sigma_{x}}(x)$. Кроме того, предполагается $I_{s}(2)$-инвариантность по отношению к некоммутативным переменным. 
В этом случае подобно тому, как из условия (31) следует равенство (30), условие (32) приводит к равенству

$$
\begin{aligned}
& \left\langle\Psi_{0}, \tilde{\varphi}^{\sigma_{1}}\left(x_{1}\right) \ldots \tilde{\varphi}^{\sigma_{i}}\left(x_{i}\right) \tilde{\psi}^{\sigma_{x}}(x) \tilde{\varphi}^{\sigma_{i+1}}\left(x_{i+1}\right) \ldots, \tilde{\varphi}^{\sigma_{n}}\left(x_{n}\right) \Psi_{0}\right\rangle= \\
& \quad=\left\langle\Psi_{0}, \tilde{\varphi}^{\sigma_{n}}\left(-x_{n}\right) \ldots \tilde{\varphi}^{\sigma_{i+1}}\left(-x_{i+1}\right) \tilde{\psi}^{\sigma_{x}}(-x) \tilde{\varphi}^{\sigma_{i}}\left(-x_{i}\right) \ldots \tilde{\varphi}^{\sigma_{1}}\left(-x_{1}\right) \Psi_{0}\right\rangle
\end{aligned}
$$

для любых $x, x_{1}, \ldots, x_{n}$. Легко видеть, что равенство (33) может быть записано в виде

$$
\left\langle\Phi, \tilde{\psi}^{\sigma_{x}}(x) \Psi\right\rangle=\left\langle\Theta \Psi, \tilde{\psi}^{\sigma_{x}}(-x) \Theta \Phi\right\rangle,
$$

где $\Phi=\tilde{\varphi}^{\sigma_{i}^{+}}\left(x_{i}\right) \ldots \tilde{\varphi}^{\sigma_{1}^{+}}\left(x_{1}\right) \Psi_{0}, \Psi=\tilde{\varphi}^{\sigma_{i+1}}\left(x_{i+1}\right) \ldots \tilde{\varphi}^{\sigma_{n}}\left(x_{n}\right) \Psi_{0}$.

Вследствие антиунитарности оператора $\Theta$ всегда

$$
\begin{aligned}
\left\langle\Phi, \tilde{\psi}^{\sigma_{x}}(x) \Psi\right\rangle & =\left\langle\tilde{\psi}^{\sigma_{x}^{+}}(x) \Phi, \Psi\right\rangle=\overline{\left\langle\Psi, \tilde{\psi}^{\sigma_{x}^{+}}(x) \Phi\right\rangle}= \\
& =\left\langle\Theta \Psi, \Theta \tilde{\psi}^{\sigma_{x}^{+}}(x) \Theta^{-1} \Theta \Phi\right\rangle .
\end{aligned}
$$

Сравнение (34) и (35) приводит к требуемому результату, поскольку $\Phi$ и $\Psi$ - произвольные базисные векторы. В свою очередь, как и в случае CPT-теоремы, условие (33) приводит к равенству (32). Далее, поскольку $\Theta$ антиунитарен, $\tilde{\psi}(x)-$ слаболокальное поле, т.е. поле, удовлетворяющее условию слабой локальной коммутативности. Более того, выполнено условие взаимной слабой локальной коммутативности. Это означает, что функции Уайтмана, содержащие любое число полей $\tilde{\varphi}\left(x_{i}\right)$ и $\tilde{\psi}\left(y_{j}\right)$, не меняются в точках Йоста при замене прямого порядка аргументов на обратный.

Наиболее важный вывод состоит в том, что условие взаимной слабой локальности транзитивно, т.е. поля $\tilde{\psi}_{1}(x)$ и $\tilde{\psi}_{2}(x)$, слаболокальные с полем $\tilde{\varphi}(x)$, слаболокальны и между собой. Действительно, согласно предыдушим рассуждениям их операторы CPT-преобразования совпадают.

Таким образом, как и в коммутативном случае, слаболокальные поля образуют класс эквивалентности Борхерса, в который входит поле $\tilde{\varphi}(x)$ и все слаболокальные с ним поля, которые при этом оказываются слаболокальными между собой. Наиболее важное свойство этих полей состоит в том, что $S$-матрица совпадает для полей, принадлежащих одному классу эквивалентности Борхерса, если совпадают их пределы при $x_{0} \rightarrow-\infty$ или $x_{0} \rightarrow+\infty$. Действительно, известно, что $S$-матрица следующим образом связана с операторами $C P T$-преобразования [3]:

$$
S=\Theta^{-1} \Theta_{\text {out }}=\Theta_{\text {in }}^{-1} \Theta,
$$

где $\Theta_{\text {in }}$ и $\Theta_{\text {out }}-$ соответствующие операторы для асимптотических полей.

Как известно [1], [3], в коммутативной теории доказано существование пределов взаимодействуюших полей при $x_{0} \rightarrow \pm \infty$ - теорема Хаага-Рюэля. Безусловно, было бы интересно получить аналог этой теоремы и в некоммутативном случае, что составит предмет отдельного исследования.

Можно показать, что и условие локальной коммутативности также транзитивно. Это означает, что два поля, локальные с полем $\tilde{\varphi}(x)$, для которого $\Psi_{0}$ - циклический вектор, взаимно локальны. Доказательство аналогично соответствующему в коммутативном случае [3]. 


\section{7. ТЕОРЕМА О СВЯЗИ СПИНА И СТАТИСТИКИ}

Теорема о связи спина и статистики для комплексного скалярного поля сводится к соответствуюшей теореме для эрмитова поля с помошью следуюшей леммы

ЛЕммА. Пусть справедливы следующие коммутационные соотношения:

$$
\begin{aligned}
{[\tilde{\varphi}(x), \tilde{\psi}(y)] } & =0, & & x \sim y, \\
\left\{\tilde{\varphi}(x), \tilde{\psi}^{+}(y)\right\} & =0, & & x \sim y .
\end{aligned}
$$

Тогда если $\tilde{\varphi}(x) \neq 0$, то $\tilde{\psi}(y) \equiv 0$.

Как и в обычном случае, предполагается, что операторы $\tilde{\varphi}(x)$ и $\tilde{\psi}(y)$ действуют на подпространстве с положительно определенной метрикой.

При доказательстве леммы сушественно используются кластерные свойства функций Уайтмана. Детальное исследование этих свойств выходит за рамки настоящей статьи. Однако вследствие условия локальной коммутативности (14) заведомо

$$
\begin{aligned}
& \left\langle\Psi_{0}, \tilde{\varphi}\left(x_{1}\right) \ldots \tilde{\varphi}\left(x_{i}\right) \tilde{\varphi}\left(x_{i+1}+\lambda a\right) \ldots, \tilde{\varphi}\left(x_{n}+\lambda a\right) \Psi_{0}\right\rangle \rightarrow \\
& \quad \rightarrow\left\langle\Psi_{0}, \tilde{\varphi}\left(x_{1}\right) \ldots \tilde{\varphi}\left(x_{i}\right) \Psi_{0}\right\rangle\left\langle\Psi_{0}, \tilde{\varphi}\left(x_{i+1}\right) \ldots \tilde{\varphi}\left(x_{n}\right) \Psi_{0}\right\rangle,
\end{aligned}
$$

если $\lambda \rightarrow \infty, a_{0}^{2}-a_{3}^{2}=-1$. Доказательство соотношения (39) сводится к соответствуюшему доказательству в коммутативном случае [1].

Для доказательства леммы достаточно заметить, что согласно (37) и (38) при $x \sim y$

$$
\begin{gathered}
\left\langle\Psi_{0}, \tilde{\varphi}^{+}(x) \tilde{\psi}^{+}(y) \tilde{\psi}(y) \tilde{\varphi}(x) \Psi_{0}\right\rangle=-\left\langle\Psi_{0}, \tilde{\varphi}^{+}(x) \tilde{\varphi}(x) \tilde{\psi}^{+}(y) \tilde{\psi}(y) \Psi_{0}\right\rangle \rightarrow \\
\rightarrow-\left\langle\Psi_{0}, \tilde{\varphi}^{+}(x) \tilde{\varphi}(x) \Psi_{0}\right\rangle\left\langle\Psi_{0}, \tilde{\psi}^{+}(y) \tilde{\psi}(y) \Psi_{0}\right\rangle
\end{gathered}
$$

если $y=x+\lambda a, \lambda \rightarrow \infty, a_{0}^{2}-a_{3}^{2}=-1$. Замечая, что исходная функция Уайтмана положительна, а последнее выражение в формуле (40) отрицательно, приходим к выводу, что выполнено одно из равенств

$$
\tilde{\varphi}(x) \Psi_{0}=0, \quad \tilde{\psi}(y) \Psi_{0}=0 .
$$

Покажем, что первое из этих равенств означает, что $\tilde{\varphi}(x) \equiv 0$, а второе - что $\tilde{\psi}(y) \equiv 0$.

Действительно, пусть, например, выполнено второе из равенств (41). Тогда вследствие условий (37) и (38)

$$
\left\langle\Psi_{0}, \tilde{\varphi}^{\sigma_{1}}\left(\tilde{x}_{1}\right) \ldots \tilde{\varphi}^{\sigma_{i}}\left(\tilde{x}_{i}\right) \tilde{\psi}(y) \tilde{\varphi}^{\sigma_{i+1}}\left(\tilde{x}_{i+1}\right) \ldots \tilde{\varphi}^{\sigma_{n}}\left(\tilde{x}_{n}\right) \Psi_{0}\right\rangle=0
$$

если $y \sim \tilde{x}_{j}$ для всех $j$. Поскольку равенство нулю функции Уайтмана в точках Йоста означает, что она тождественно равна нулю, то

$$
\left\langle\Psi_{0}, \tilde{\varphi}^{\sigma_{1}}\left(x_{1}\right) \ldots \tilde{\varphi}^{\sigma_{i}}\left(x_{i}\right) \tilde{\psi}(y) \tilde{\varphi}^{\sigma_{i+1}}\left(x_{i+1}\right) \ldots \tilde{\varphi}^{\sigma_{n}}\left(x_{n}\right) \Psi_{0}\right\rangle=0
$$


для любого $x_{i}$. Учитывая, что $\Psi_{0}$ - циклический вектор для поля $\tilde{\varphi}(x)$, получим, что $\tilde{\psi}(y) \equiv 0$. Те же самые рассуждения применимы и к $\tilde{\varphi}(x)$. Поскольку по предположению $\tilde{\varphi}(x) \neq 0$, утверждение леммы доказано.

Далее применим лемму к случаю $\tilde{\psi}(x)=\tilde{\varphi}(x)$ и докажем, что если

$$
\left\{\tilde{\varphi}(x), \tilde{\varphi}^{+}(y)\right\}=0, \quad x \sim y
$$

то $\tilde{\varphi}(x) \equiv 0$. Для этого рассмотрим функции

$$
\begin{aligned}
& W_{1}(x-y)=\left\langle\Psi_{0}, \tilde{\varphi}(x) \tilde{\varphi}^{+}(y) \Psi_{0}\right\rangle, \\
& W_{2}(x-y)=\left\langle\Psi_{0}, \tilde{\varphi}^{+}(x) \tilde{\varphi}(y) \Psi_{0}\right\rangle .
\end{aligned}
$$

Применяя к ним рассуждения, приведенные в работе [27], придем к выводу, что условие (44) означает, что $\tilde{\varphi}(x) \Psi_{0}=0$, т.е. $\tilde{\varphi}(x) \equiv 0$.

\section{8. ЗАКЛЮЧЕНИЕ}

Проведенное исследование показывает, что если в трансляционной инвариантной теории существует аналог условий локальной коммутативности (14) и спектральности (15) то $S O(1,1) \otimes I_{s}(2)$-инвариантности теории достаточно для вывода основных результатов аксиоматического подхода. Физически интересным вариантом такой теории является некоммутативная квантовая теория поля, в которой время коммутирует с пространственными координатами.

\section{Список литературы}

[1] P. Cтритер, A. Вайтман. РCT, спин и статистика и все такое. М.: Наука, 1966.

[2] $P$. Йост. Общая теория квантованных полей. М.: Мир, 1967.

[3] Н. Н. Боголюбов, А. А. Логунов, И. Т. Тодоров. Основы аксиоматического подхода в квантовой теории поля. М.: Наука, 1969.

[4] R. Haag. Local Quantum Physics. Berlin: Springer, 1996.

[5] G. Morchio, F. Strocchi. Ann. Inst. H. Poincaré A. 1980. V. 33. P. 251.

[6] F. Strocchi. Selected Topics on the General properties of Quantum Field Theory. Singapore: World Scientific, 1993.

[7] А.А. Логунов, М.А. Мествиришвили, О. А. Хрусталев. ЭЧАЯ. 1972. Т. 3. С. 3.

[8] H.S. Snyder. Phys. Rev. 1947. V. 71. P. 38.

[9] A. Connes. Noncommutative Geometry. New York: Acad. Press, 1994.

[10] S. Doplicher, K. Fredenhagen, J. E. Roberts. Phys. Lett. B. 1994. V. 331. P. 39; Commun. Math. Phys. 1995. V. 172. P. 187.

[11] N. Seiberg, E. Witten. JHEP. 1999. V. 9909. P. 32; hep-th/9908142.

[12] M. R. Douglas, N. A. Nekrasov. Rev. Mod. Phys. 2001. V. 73. P. 977; hep-th/0106048.

[13] R. J. Szabo. Phys. Rep. 2003. V. 378. P. 207; hep-th/0109162.

[14] J. Gomis, T. Mehen. Nucl. Phys. B. 2000. V. 591. P. 265; hep-th/0005129.

[15] N. Seiberg, L. Susskind, N. Toumbas. JHEP. 2000. V. 0006. P. 044; hep-th/0005015; L. Álvarez-Gaumé, J.L.F. Barbon. Int. J. Mod. Phys. A. 2001. V. 16. P. 1123; hep-th/0006209.

[16] M. Chaichian, K. Nishijima, A. Tureanu. Phys. Lett. B. 2003. V. 568. P. 146; hep-th/0209008.

[17] O. Aharony, J. Gomis, T. Mehen. JHEP. 2000. V. 0009. P. 023; hep-th/0006236. 
[18] Yi Liao, K. Sibold. Phys. Lett. B. 2002. V. 549. P. 352; hep-th/0209221.

[19] M. Chaichian, M. Mnatsakanova, A. Tureanu, Yu. Vernov. Nucl. Phys. B. 2003. V. 673. P. 476; hep-th $/ 0306158$.

[20] Ю. С. Вернов, М. Н. Мнацаканова. ТМФ. 2004. Т. 139. С. 3.

[21] M. Chaichian, A. Tureanu. Jost-Lehmann-Dyson representation and Froissart-Martin bound in quantum field theory on noncommutative space-time. hep-th/0403032.

[22] Ю. С. Вернов, М. М. Мнацаканова. ТМФ. 2005. Т. 142. С. 388; hep-th/0403033.

[23] J. Mund, B. Schroer, J. Yngvason. Phys. Lett. B. 2004. V. 596. P. 156; math-ph/0402043.

[24] S. Doplicher. Spacetime and fields, a quantum texture. In: New Developments in Fundamental interaction Theories. 37th Karpacz Winter School of Theoretical Physics, Parpacz, Poland, Febr. 6-15, 2001. AIP Conf. Proc. V. 589. Eds. J. Lukierski, J. Rembielinski. Melville, NY: AIP, 2001. P. 204; hep-th/0105251.

[25] R. Brunetti, K. Fredenhagen, R. Verch. Commun. Math. Phys. 2003. V. 237. P. 31; math-ph/0112041.

[26] L. Álvarez-Gaumé, M. A. Vázquez-Mozo. Nucl. Phys. B. 2003. V. 668. P. 293; hep-th/0305093.

[27] M. Chaichian, M. N. Mnatsakanova, K. Nishijima, A. Tureanu, Yu.S. Vernov. Towards an axiomatic formulation of noncommutative quantum field theory. hep-th/0402212.

[28] L.Álvarez-Gaumé, J. L. F. Barbon, R. Zwicky. JHEP. 2001. V. 0105. P. 057; hep-th/0103069.

[29] D. H. T. Franco. On the Borchers class of a non-commutative field. hep-th/0404029.

[30] О. Штейнман. Метод возмущений в аксиоматической теории поля. М.: Мир, 1974.

[31] B. C. Владимиров. Методы теории функций многих комплексных переменных. М.: Наука, 1964.

Зав. редакцией С. В. Сушко

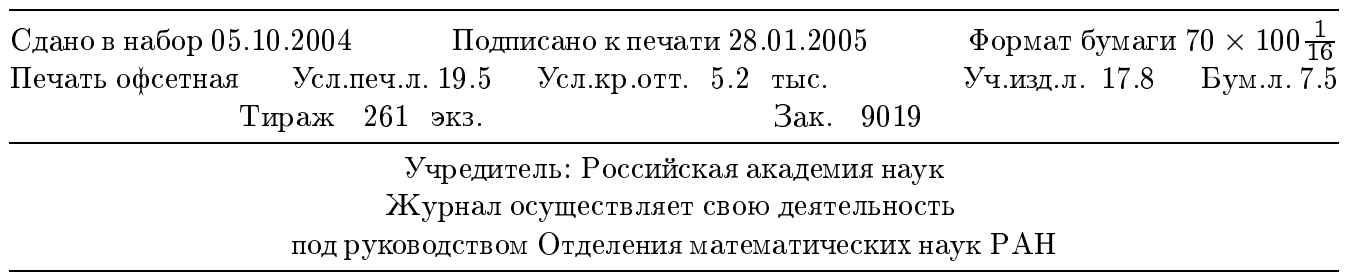

А ДРЕС ИЗДАТЕЛЯ: 117997, Москва, Профсоюзная ул., 90

АдРЕс РЕДАКциИ: 119991, Москва, ул. Губкина, 8, тел. 135-20-19,

e-mail: tmph@mi.ras.ru

Отпечатано в ППП «Типография «Наука», 121099, Москва, Шубинский пер., 6 